\title{
Erratum to: Features of Heat Transfer on a Permeable Surface in a Compressible-Gas Flow
}

\author{
Academician A. I. Leont'ev ${ }^{a, b}$, V. G. Lushchik ${ }^{a}$, and M. S. Makarova ${ }^{a, *}$ \\ Received December 24, 2018
}

DOI: $10.1134 / \mathrm{S} 1028335819010117$

The list of affiliations should read as follows:

${ }^{a}$ Moscow State University, Research Institute of Mechanics, Moscow, 119192 Russia

${ }^{b}$ Bauman Moscow State Technical University, Moscow, 105005 Russia

Page 372, left column, line 4 from top should read: $\gamma=1.667$

Page 373, caption to Fig. 3, line 2 should read:

$\operatorname{Pr}=3$

Page 374, section ACKNOWLEDGMENTS, line 2 should read:

project no. 14-19-00699

The original article can be found online at

https://doi.org/10.1134/S1028335818090033

\footnotetext{
${ }^{a}$ Moscow State University, Research Institute of Mechanics, Moscow, 119192 Russia

${ }^{b}$ Bauman Moscow State Technical University, Moscow, 105005 Russia

*e-mail: april27_86@mail.ru
} 